Revista Destaques Acadêmicos, Lajeado, v. 10, n. 4, 2018. ISSN 2176-3070

DOI: http://dx.doi.org/10.22410/issn.2176-3070.v10i4a2018.2044

http://www.univates.br/revistas

\title{
SISTEMA DE GERÊNCIA E DOCUMENTAÇÃO PARA REDES PON (FTTX)
}

\author{
Leandro Carlesso ${ }^{1}$, Edson Moacir Ahlert ${ }^{2}$
}

Resumo: A evolução em telecomunicações com o uso intenso de fibra óptica acompanhando a expansão tecnológica e a demanda de transmissão de dados tornouse uma necessidade na atualidade. Novas estratégias vêm sendo adotadas para as redes de acesso e, dentre as mais proeminentes, estão as Redes Ópticas Passivas (PON) no contexto de FTTH (Fiber to the Home). Baseadas em fibra óptica e utilizando divisores de potência não alimentados por energia elétrica, essas redes são uma alternativa economicamente viável e cada vez mais comum. Este trabalho teve como objetivo construir uma ferramenta tecnológica que dê apoio aos funcionários de um provedor de Internet, e consiste em uma ferramenta de gerência e documentação de uma rede FTTX, baseada em mapa, com cadastros de caixas de atendimento, cadastro de clientes, entre outras funções. A ferramenta proposta contribui com a documentação da rede, agilidade na instalação e manutenção, com informações de CTOs (caixas de terminação ópticas) ou caixa de atendimento ao cliente próximas, além de manter um inventário atualizado e gerência facilitada dos recursos da rede óptica.

Palavras-chave: Redes Ópticas. PON. FTTH. Gerência de Redes.

\section{INTRODUÇÃO}

A Internet ou rede mundial de computadores foi inicialmente criada na guerra fria, entre as décadas de 1970 e 1980 com objetivos militares, para se constituir numa forma de comunicação robusta e sem falhas. Um grande marco da Internet foi em 1990, quando foi criado protocolo Hypertext Transfer Protocol (HTTP) e a linguagem Hypertext Markup Language (HTML), que juntas tornaram-se a World Wide Web (WWW), ou seja, a "Rede de Alcance Mundial".

Atualmente, a tecnologia que mais tem se destacado como a melhor alternativa para suprir as crescentes demandas em redes de acesso de telecomunicações é a fibra óptica, no conceito Fiber To The Home (FTTH), ou

1 Graduado em Engenharia da Computação pela Univates.

2 Professor da Univates. Mestre em Ambiente e Desenvolvimento. 
seja, a fibra óptica chegando até as residências. Sua maior vantagem é a baixa latência, maior velocidade e confiabilidade, chegando à faixa dos gigabits por segundo.

A necessidade de velocidades cada vez maiores no acesso à Internet se dá pelos inúmeros serviços disponibilizados, tais como acesso a sistemas e serviços de bancos de dados e telefonia, importantes para as empresas; rede de sensores e monitoramento, importante para a segurança pública e indústrias em geral; acesso a vídeos, redes sociais e jogos, demanda de usuários domésticos; além de compras online, home banking, e uma infinidade de outros serviços e negócios.

Devido a essa demanda os provedores de serviço estão investindo alto em novas redes FTTH, e uma grande preocupação é sobre a gerência e documentação destas redes, com vistas também a facilitar uma posterior manutenção desta rede, pois não há muitos softwares no mercado e muitos deles exigem um alto investimento e muitas vezes possuem pouca integração com os sistemas do provedor.

Partindo desta premissa, este trabalho teve como objetivo desenvolver um sistema para controle de redes ópticas FTTH, baseado em mapas, propondo uma ferramenta para as atividades de gestão, controle e manutenção de redes ópticas passivas.

A ferramenta proposta pretende contribuir com a documentação da rede, agilidade na instalação aos clientes, na facilidade de conhecimento da rede e inclusão de novos clientes via aplicativo, por meio de dispositivos móveis, e trará informações de onde estão as caixas de atendimento próximas e se há vagas disponíveis para novos clientes nessas caixas.

\section{REDES ÓPTICAS PASSIVAS}

Nesta seção serão abordadas as referências teóricas sobre fibras ópticas, suas características e aplicações. Ademais serão também conceituadas características de projetos e gerência de redes ópticas da atualidade.

\subsection{Redes Ópticas Passivas}

Uma conexão de fibra óptica tem baixa perda entre seu transmissor e receptor, além de conseguir transmitir sinais analógicos ou digitais, o sinal é convertido de elétrico para óptico por meio de um conversor de mídia, em uma transmissão ponto a ponto, ou de uma (OLT) Optical Line Terminal, se utilizar FTTH. A conversão eletro-óptica e vice-versa é necessária, pois após a recepção, normalmente precisamos entregar esse sinal a um switch ou a uma placa de rede (MARIN, 2015).

Até hoje, a principal aplicação das fibras ópticas estão em redes de longas distâncias, interligações de serviços de telefonia e backbone de Internet, 
mas com o aumento das velocidades entregues até os clientes, as redes ópticas passivas estão tomando um lugar importante na distribuição de Internet até o cliente, conceito este chamado de FTTH (KEISER, 2014).

Há outras derivações do nome, um dos mais comuns é o FTTx, ou "Fibra até $X$ " onde " $X$ " pode ser "Home" ou "Apartment", nestas três modalidades a fibra vai da central até o usuário final. Já nas modalidades FTTN, fibra até um nó, que pode ser um poste ou qualquer outro ponto, FTTC onde a fibra chega somente até um armário de rua e FTTB fibra chegando até o prédio, após esses pontos o sinal pode ser distribuído por outra tecnologia como DSL ou cabo de par trançado ou a que for conveniente para a operadora (FRENZEL, 2013).

A inovação nas redes ópticas passivas veio por conta da remoção dos repetidores de sinal, que são um incômodo para manutenção da rede e consomem energia, e em lugar destes foram utilizados elementos passivos, que não necessitam de energia. Daí vem o nome da tecnologia, redes ópticas passivas ou PON.

Figura 1 - Estrutura de Redes FTTH

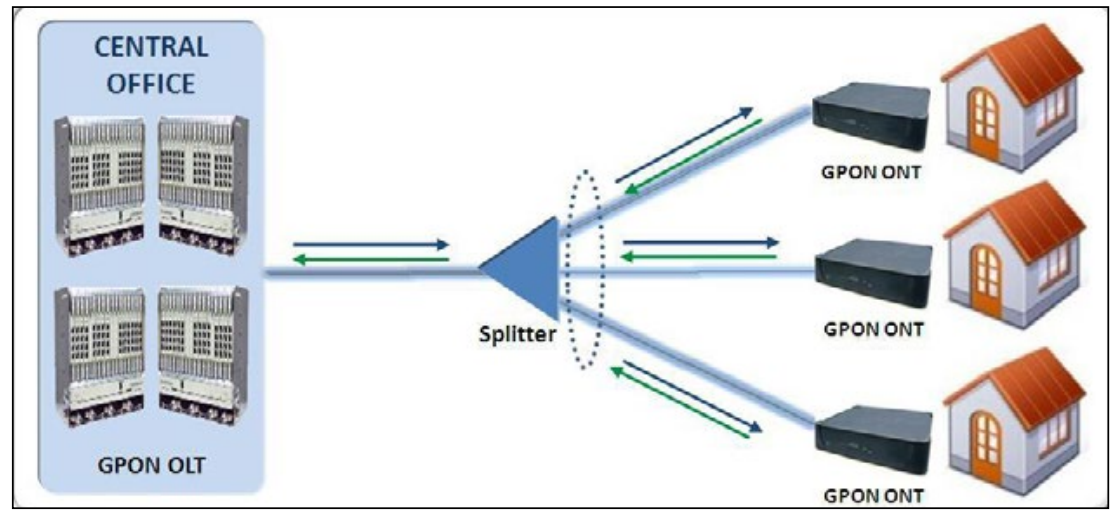

Fonte: Furukawa (2017).

Independente das variações dos nomes que relatam a FTTX, os componentes principais da tecnologia serão compostos por uma rede de fibra óptica monomodo, equipamentos para as centrais de assinantes OLT e elementos passivos que estão no meio do caminho, denominados splitters, conforme Figura 1 (FURUKAWA, 2017).

Estes elementos passivos utilizados nestes enlaces são os divisores ou combinadores de sinais, utilizando lasers de alta potência e cabos com pouca perda de sinal consegue-se chegar até $20 \mathrm{~km}$ em uma rede PON sem qualquer tipo de amplificação do sinal e é por esse motivo que a rede PON tem conquistado o mercado (FRENZEL, 2013). 
Para Keiser (2014) as redes PON devem dominar o mercado de telecomunicações, principalmente nas aplicações de dados e telefonia pela Internet, bem como serviços outros a partir da Internet, como a IPTV. A (IPTV) Internet Protocol Television ou (TVIP) Televisão por IP é um novo método de transmissão de sinais televisivos. Assim como o Voz sobre IP (VOIP), o (IPTV) usa o protocolo IP Internet Protocol como meio de transporte do conteúdo.

Atualmente o padrão mais comum de redes PON é o GPON, com capacidades Gigabit, definido pela séria de recomendações da ITU-T no G.984. Dando uma visão geral de alto nível dos componentes GPON e sua estrutura de referência, a GPON também definiu uma única fibra ou fibra dupla, sua capacidade é upstream é $2488.32 \mathrm{Mbps}$ (arredondando para 2,5 Gbps) e downstream $1244.16 \mathrm{Mbps}$ (arredondando para 1,25 Gbps). Assim, suas taxas de dados são elevadas e podem ser simétricas ou assimétricas (ITU-T, 2008).

\subsection{Elementos do sistema de comunicação Óptica Passiva}

Para Keiser (2014), uma rede PON é estrutura a partir dos equipamentos envolvidos na rede, desde a central de comunicação, até o equipamento do cliente. Vamos apresentar agora os principais elementos que fazem essa rede funcionar.

Um dos principais elementos de uma rede PON é o splitter, também chamado de divisor óptico, ele fica entre a OLT e a ONU, instalado dentro da (CTO) caixa de terminação óptica ou da (CEO) caixa de emenda óptica. Ele é um elemento passivo, que não necessita alimentação por energia elétrica nem refrigeração, e tem por finalidade dividir o sinal óptico de entrada em múltiplas saídas que se conectaram às ONU dos clientes.

As caixas de atendimento ao cliente ou CTO é a caixa que fica instalada no poste, onde recebe o cabo primário adicionado um splitter óptico que recebe em suas saídas os cabos drop, para acesso final ao cliente em redes FTTH (FURUKAWA, 2017).

O equipamento que distribui o sinal para os splitters e as ONU's é chamado de (OLT) - Optical Line Terminal ou Terminal de Linha Óptico, ele faz o gerenciamento de usuários e realiza as tarefas de controle de acesso, controle de banda, disponibilização de serviços entre outros, ele recebe os dados por meio de suas portas de uplink e os transmite para suas portas PON, que pode receber até 64 clientes em cada porta com uma banda compartilhada 2,5 Gbps utilizando o padrão (GPON) Gigabit PON, padrão definido pelo ITU-T (ITU-T, 2008).

O equipamento que fica na casa do cliente chama-se (ONU) Optical Network Unit ou (ONT) Optical Network Terminal e é capaz de transformar o sinal óptico que chega à porta $\mathrm{PON}$ em sinal elétrico, que sai na porta da rede local, além de funções de roteamento, DHCP, entre outras (FURUKAWA 2017). 


\subsection{Projeto de Rede FTTX}

Segundo Levay (2013), um projeto PON deve ser iniciado em relação a um mapa, pois o projeto é considerado com base na geografia e na infraestrutura disponível no local, assim será possível dizer em quais pontos a OLT, os splitters e as ONUs podem ser instalados.

Atualmente, temos diversos sistemas digitais de mapeamento de cidades com informações de ruas, avenidas, esquinas, pontos de interesses, visualização de imagens de satélite, vista da rua, prédios em $3 \mathrm{D}$, tudo isso baseado em sistema de geolocalização (LEVAY, 2013).

O projeto de uma rede FTTX leva em conta muitas variáveis. Lafata (2013) defende que um software que faça a simulação do projeto consegue chegar a dar uma estimativa de onde é o melhor local para ficar a OLT e onde deixar as CTOs para obter o menor custo em infraestrutura.

Utilizando um software que faça a simulação do projeto, o mesmo deve ser revisado por um engenheiro para prever os cálculos e ver se está tudo em conformidade, pois uma rede mal projetada pode se tornar um grave problema e com grande chance de ter que ser refeita.

Outro ponto muito importante do padrão GPON é a quantidade de equipamentos que cada porta GPON, suporta a razão de 1:128, ou seja, podemos colocar 128 ONUs para cada porta GPON, o padrão dá suporte a QoS, segurança, entre outras funcionalidades, o que nenhum dos padrões anteriores suportavam, isso torna o padrão muito superior aos outros.

\section{PROCEDIMENTOS METODOLÓGICOS}

Nesta seção são abordados o conjunto de ferramentas, métodos e tecnologias utilizadas no desenvolvimento do software proposto e as suas principais características. O objetivo foi desenvolver um sistema para controle de redes ópticas FTTX, baseado em mapas, propondo uma ferramenta para atividades de gestão, controle e manutenção de redes ópticas passivas.

A ferramenta proposta pretende contribuir com a documentação da rede, agilidade na instalação aos clientes, pela facilidade de conhecimento da rede e inclusão de novos clientes, via aplicativo de dispositivos móveis, e trará informações de onde estão as caixas de atendimento próximas e se há vagas disponíveis para novos clientes nessas caixas.

De forma resumida o projeto iniciou com uma pesquisa exploratória delimitando o problema a ser resolvido e quais as dificuldades e delimitações. Então foi realizado um levantamento bibliográfico com base no problema e conceituados os temas e tecnologias que seriam tratados ao longo do projeto e após levantados os requisitos do sistema a ser desenvolvido e posterior desenvolvimento e implementação. 
Apresenta-se a seguir, o Quadro1, com a descrição da metodologia utilizada neste trabalho, com o objetivo de expor os caminhos que foram percorridos não só no levantamento dos dados do estudo, como também na forma de fazê-lo. Os dados pesquisados, segundo os métodos adotados e em articulação ao referencial teórico, pretendem dar explicações com o intuito de responder aos objetivos propostos.

Quadro 1 - Metodologia da Pesquisa

\begin{tabular}{|l|l|l|}
\hline $\begin{array}{l}\text { Quanto à } \\
\text { natureza }\end{array}$ & Aplicada & $\begin{array}{l}\text { Busca gerar conhecimento para aplicação prática e } \\
\text { dirigida à solução de problemas específicos. }\end{array}$ \\
\hline $\begin{array}{l}\text { Quanto à } \\
\text { abordagem do } \\
\text { problema }\end{array}$ & $\begin{array}{l}\text { Quali- } \\
\text { quantitativa }\end{array}$ & $\begin{array}{l}\text { O levantamento e coleta de dados sobre a aplicação } \\
\text { desenvolvida valeu-se de entrevistas e percepção } \\
\text { dos usuários do sistema. }\end{array}$ \\
\hline \multirow{2}{*}{$\begin{array}{l}\text { Quanto aos } \\
\text { objetivos }\end{array}$} & Exploratória & $\begin{array}{l}\text { Objetiva um estudo de caso no próprio ambiente e } \\
\text { contexto institucional. }\end{array}$ \\
\cline { 2 - 3 } & Descritiva & $\begin{array}{l}\text { Realizada por instrumentos padronizados para } \\
\text { a coleta de dados, formulação da proposta, } \\
\text { implementação, testes e validação. }\end{array}$ \\
\hline $\begin{array}{l}\text { Quanto aos } \\
\text { procedimentos } \\
\text { técnicos }\end{array}$ & $\begin{array}{l}\text { Pesquisa } \\
\text { bibliográfica }\end{array}$ & $\begin{array}{l}\text { Para consolidar o embasamento teórico do estudo, } \\
\text { pesquisa bibliográfica forneceu os subsídios } \\
\text { necessários para o trabalho. }\end{array}$ \\
\cline { 2 - 3 } & $\begin{array}{l}\text { Pesquisa } \\
\text { documental }\end{array}$ & $\begin{array}{l}\text { Utilizando os registros da instituição, para obter } \\
\text { informações para atender os objetivos propostos. }\end{array}$ \\
\hline
\end{tabular}

Fonte: Do autor (2017), baseado em Gil (2010) e Chemin (2015).

A metodologia de trabalho está dividida em cinco etapas interdependentes e que sucedem de forma cronológica conforme descrito no fluxograma da Figura 2, que apresenta, de forma resumida, as etapas do trabalho.

Figura 2 - Fluxograma do desenvolvimento da pesquisa

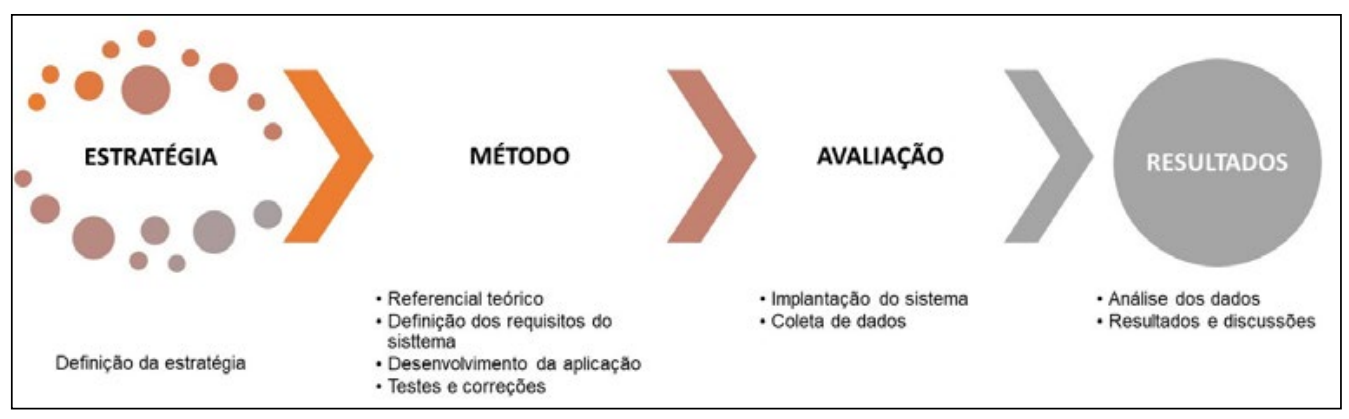

Fonte: Elaborado pelo autor (2017). 
No Quadro 2 temos as principais ferramentas utilizadas no desenvolvimento da solução, todas as ferramentas são gratuitas, mas algumas têm recursos limitados, somente disponibilizados ao adquirir uma licença de utilização, incluindo aplicativos de mapas, aplicativos web, bancos de dados, linguagens de programação, frameworks e plataforma para aplicações móveis.

Quadro 2 - Ferramentas e aplicativos utilizados no desenvolvimento do software

\begin{tabular}{|l|l|}
\hline $\begin{array}{l}\text { Ferramenta } \\
\text {-Aplicativo }\end{array}$ & Descrição \\
\hline GOOGLE & $\begin{array}{l}\text { A Google Maps API permite exibir um mapa Google no aplicativo } \\
\text { Android e esses mapas têm a mesma aparência que os mapas } \\
\text { visualizados no aplicativo Google Maps for Mobile (GMM) e a API } \\
\text { expõe muitos dos mesmos recursos (GOOGLE, 2017). }\end{array}$ \\
\hline JavaScript & $\begin{array}{l}\text { JavaScript é uma linguagem de programação que permite } \\
\text { implementar funcionalidades mais complexas em páginas web. } \\
\text { Ela permite fazer com que uma página web mostre, em tempo } \\
\text { real, conteúdos atualizados, ou mapas interativos, ou animações } \\
\text { gráficas em 2D/3D, ou vídeos em movimento (COSTA, 2007). }\end{array}$ \\
\hline Android & $\begin{array}{l}\text { O Android é a primeira plataforma para aplicações móveis } \\
\text { com código aberto (open-source), e isso representa uma grande } \\
\text { vantagem devido a diversos programadores que poderão dar } \\
\text { sua contribuição para melhorar o sistema. Para os fabricantes } \\
\text { isso é melhor ainda, por poderem utilizar esse sistema em seu } \\
\text { dispositivo, sem precisar pagar por isso (LECHETA, 2014). }\end{array}$ \\
\hline Bootstrap & $\begin{array}{l}\text { O Apache Cordova é um framework desenvolvido em HTML5, } \\
\text { CSS3 e JavaScript, sendo que seu código é transformado } \\
\text { para dispositivos móveis utilizando plataformas cruzadas } \\
\text { (CORDOVA, 2017). }\end{array}$ \\
\hline Cordova & $\begin{array}{l}\text { O AngularJS permite aplicar práticas bem conhecidas e testadas } \\
\text { na parte do back-end, e consegue uma produção acelerada na } \\
\text { parte do front-end, além de uma estrutura escalável para projetos } \\
\text { complexos, e a melhor parte é que é tudo feito em JavaScript } \\
\text { e HTML puro, o que é muito bom, pois não há necessidade de } \\
\text { aprender uma nova linguagem (SESHADRI; GREEN, 2008). }\end{array}$ \\
\hline $\begin{array}{l}\text { Bootstrap é um framework de código aberto (open-source), que } \\
\text { ajuda no desenvolvimento do front-end ele contém CSS, botões, } \\
\text { ícones e outros componentes embutidos. Suas funções foram } \\
\text { desenvolvidas em JavaScript e permite criar sites robustos e } \\
\text { responsivos, sendo totalmente personalizável (SPURLOCK, 2013). }\end{array}$ \\
\hline Angulars
\end{tabular}




\begin{tabular}{|l|l|}
\hline $\begin{array}{l}\text { Ferramenta } \\
\text {-Aplicativo }\end{array}$ & Descrição \\
\hline MySQL & $\begin{array}{l}\text { O MySQL é um sistema de gerenciamento de banco de dados } \\
\text { (SGBD), que utiliza a linguagem SQL (Linguagem de Consulta }\end{array}$ \\
$\begin{array}{l}\text { Estruturada, do inglês Structured Query Language) como } \\
\text { interface. É atualmente um dos sistemas de gerenciamento de } \\
\text { bancos de dados mais populares (COSTA, 2007). }\end{array}$ \\
\hline
\end{tabular}

Fonte: Do Autor (2017).

\section{DESENVOLVIMENTO DO SISTEMA}

Esta seção apresenta a solução desenvolvida pelo autor para auxílio a provedores de serviço de telecomunicações, para controle de redes ópticas FTTX, baseado em mapas, propondo uma ferramenta para as atividades de gestão, controle e manutenção de redes ópticas passivas. O texto está organizado em levantamento de requisitos, detalhes das funções e testes da ferramenta.

\subsection{Solução desenvolvida}

Para dar mais agilidade e suporte aos funcionários de um provedor de internet foi desenvolvido uma ferramenta chamada PonAdmin que consiste em um sistema web utilizado para gerência e um aplicativo mobile utilizado pelos funcionários, o sistema é baseado em mapa, com cadastro de caixas de distribuição, cadastro de clientes e documentação de redes FTTH, auxiliando nas atividades de gestão, controle e manutenção de redes ópticas passivas.

A ferramenta contribui com a documentação da rede, agilidade na instalação nos clientes, devido à facilidade de conhecimento da rede e inclusão de novos clientes via aplicativo de dispositivos móveis, e trará informações de onde estão as caixas de atendimento próximas e se há vagas disponíveis para novos clientes.

Os dados mantidos pelo sistema proposto necessitam ser armazenados em um sistema gerenciador de banco de dados (SGBD). O SGBD escolhido para o projeto foi o MySQL devido a sua confiabilidade, facilidade e desempenho comprovado mundialmente, os dados foram armazenados em 8 tabelas, o modelo entidade relacionamento facilita a compreensão da estrutura e dos relacionamentos que cada tabela deve conter, a tabela pode ser observada na Figura 3.

O aplicativo mobile tem a mesma base do sistema Web, mas sua principal utilização é o cadastro de clientes e visualização das caixas de atendimento, para funcionamento do aplicativo o dispositivo deve estar conectado à internet e com o GPS ativado. 
Figura 3 - Modelo entidade relacionamento

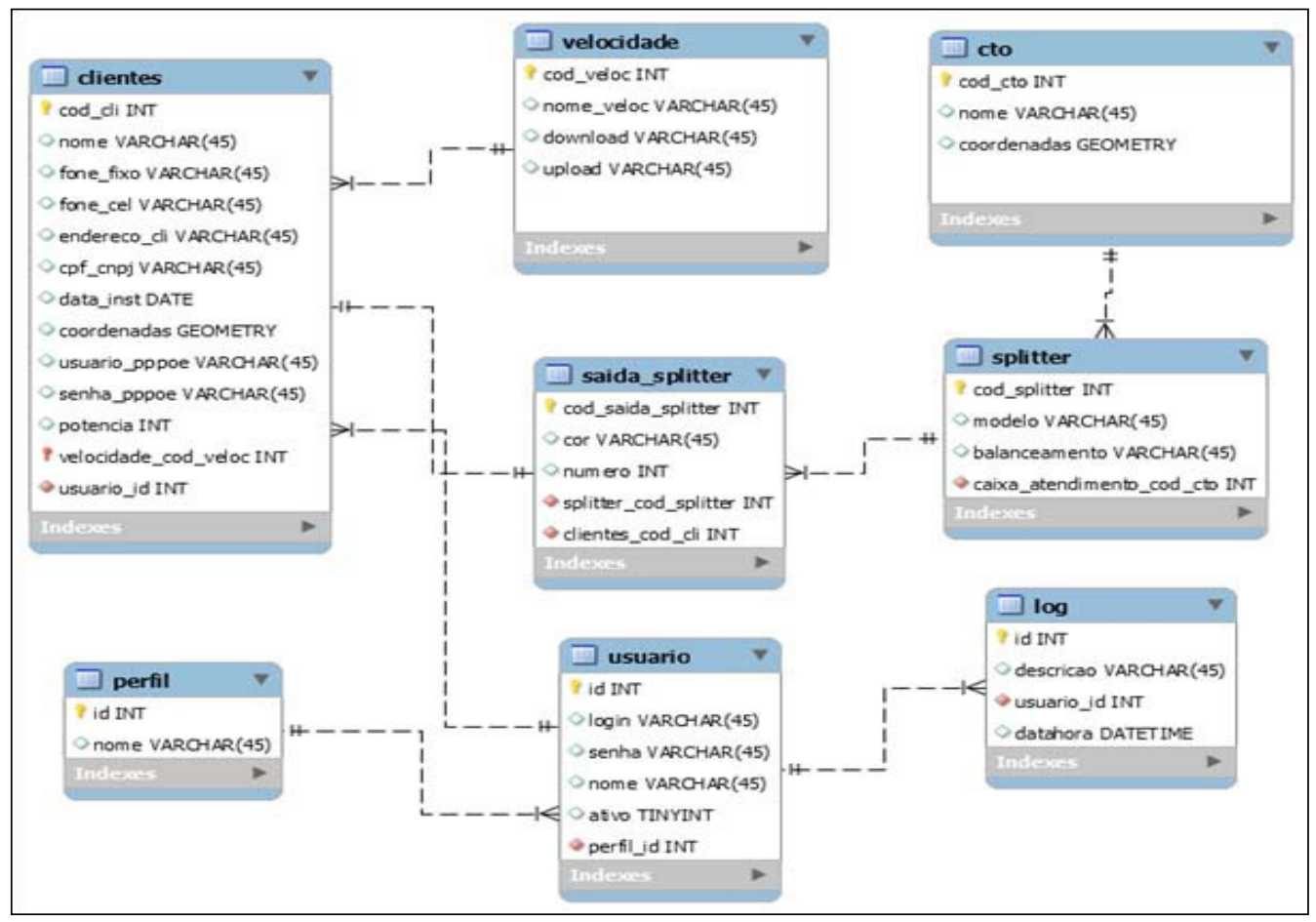

Fonte: Elaborado pelo autor (2017).

\subsection{Interface do sistema}

Apresentamos nesta seção a interface do sistema desenvolvido, para melhor compreensão e demonstração dos recursos do aplicativo.

A tela de acesso ao sistema tem por objetivo dar acesso aos usuários permitidos via login e senha previamente cadastrados. Após o login ser feito no sistema, a próxima tela que o usuário tem acesso é o menu do sistema, ele traz as opções em que cada perfil tem acesso, a Figura 4(A) mostra o menu do sistema web, com o usuário Gerência, já a Figura 4(B) mostra o aplicativo mobile com o usuário Instalador.

O menu do sistema está separado nos seguintes itens:

1. Mapa: Visualização das caixas de atendimento e clientes distribuídos no mapa em suas posições, os usuários têm informações dos clientes conectados em cada caixa de atendimento ao clicar nela;

2. Cadastro de Cliente: $\mathrm{O}$ cadastro do cliente armazena as informações pessoais e as informações e sobre a conexão do cliente, guarda as informações e cadastrada no servidor Radius da empresa; 
3. Cadastro de CTO: O cadastro de CTO ou caixa de atendimento ao cliente armazena a posição geográfica e o nome que o utilizador designar a ela;

4. Cadastro de Splitter: O cadastro de splitter guarda a informação de qual splitter foi instalado em cada caixa de atendimento;

5 a 8. Consultas: As consultas são apenas uma busca ao banco de dados trazendo as informações já cadastradas, cada consulta condiz com o seu cadastro;

9. Logs: A guia de consulta de logs busca as informações de o que cada usuário fez no sistema, inclusão, exclusão, alteração, data e a hora.

Figura 4 - Menu do sistema web (A) e menu mobile (B)
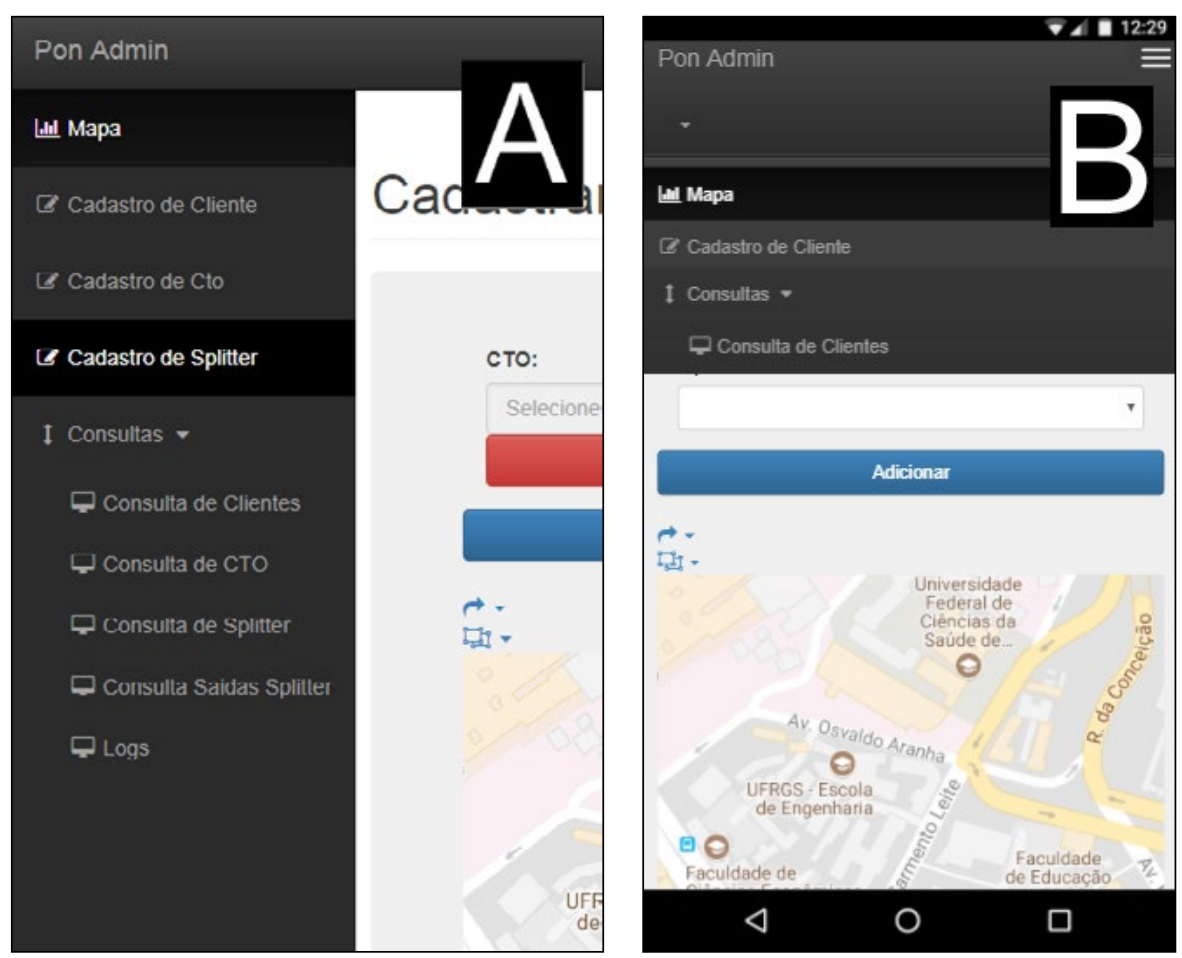

Fonte: Elaborado pelo autor (2017).

O cadastro de caixa de atendimento tem como requisito obrigatório a inserção de um nome e das coordenadas, no caso da utilização do aplicativo mobile as coordenadas já são preenchidas automaticamente com a posição GPS do smartphone, no caso do aplicativo web ou se a posição do smartphone não for a que se deseja, surge um campo com auto-complete do endereço. Ao selecionar o endereço ele é mostrado no mapa com uma marcação, que pode ser alterada gerando assim a posição desejada no mapa e assim é possível inserir no sistema. 
O cadastro de um splitter precisa informar a caixa de atendimento que ele será instalado e seu modelo, para selecionar a caixa de atendimento há um botão Pesquisar após clicar é aberto um mapa com as caixas de atendimento cadastradas ao clicar em uma delas o seu código é preenchido no campo CTO.

Um dos pontos mais importantes deste projeto é o cadastro de clientes, todos os outros cadastros dão suporte a ele, a cada nova inclusão de cliente ele armazena todas as informações no sistema.

Ao iniciar o cadastro o sistema já preenche automaticamente alguns campos, outros são requisitados ao usuário para inclusão de um novo cliente, solicitando o preenchimento dos campos Nome, Telefone, CPF/CNPJ, Data Instalação, Usuário PPPoE, Senha PPPoE, Coordenadas GPS, CTO, Saída Splitter e Velocidade.

Após a validação dos dados o sistema cadastra o cliente no banco de dados da aplicação, e então gera uma conexão via SSH ao servidor Radius da empresa, que cadastra as informações do PPPoE, a Figura 5 apresenta os dados já inseridos no servidor Radius da empresa. Assim, o instalador após ter feito o cadastro do cliente pode instalar a ONU no local e informar o usuário e senha PPPoE que foi recém cadastrado.

Figura 5 - Log do servidor Radius do cliente cadastrado

\begin{tabular}{|c|c|}
\hline Terminal & $\square \times$ \\
\hline 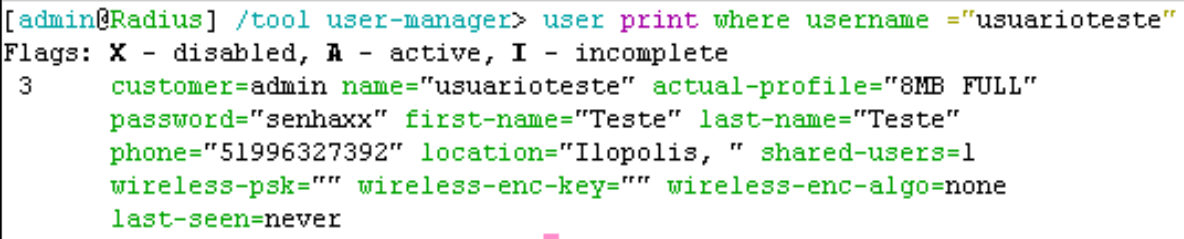 & 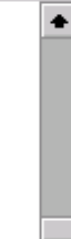 \\
\hline [admin[Radius] /tool user-manager> & \\
\hline
\end{tabular}

Fonte: Elaborado pelo autor (2017).

O processo de inclusão de um novo cliente se altera de complexo para descomplicado e acessível, pois em um único cadastro o sistema tem todos os dados da instalação do cliente.

A Figura 6 mostra como funciona o cadastro do cliente do provedor sem utilização do sistema, é necessário mais uma pessoa envolvida no cadastro para ter acesso aos sistemas internos. 
Figura 6 - Cadastro do cliente (Sem Sistema)

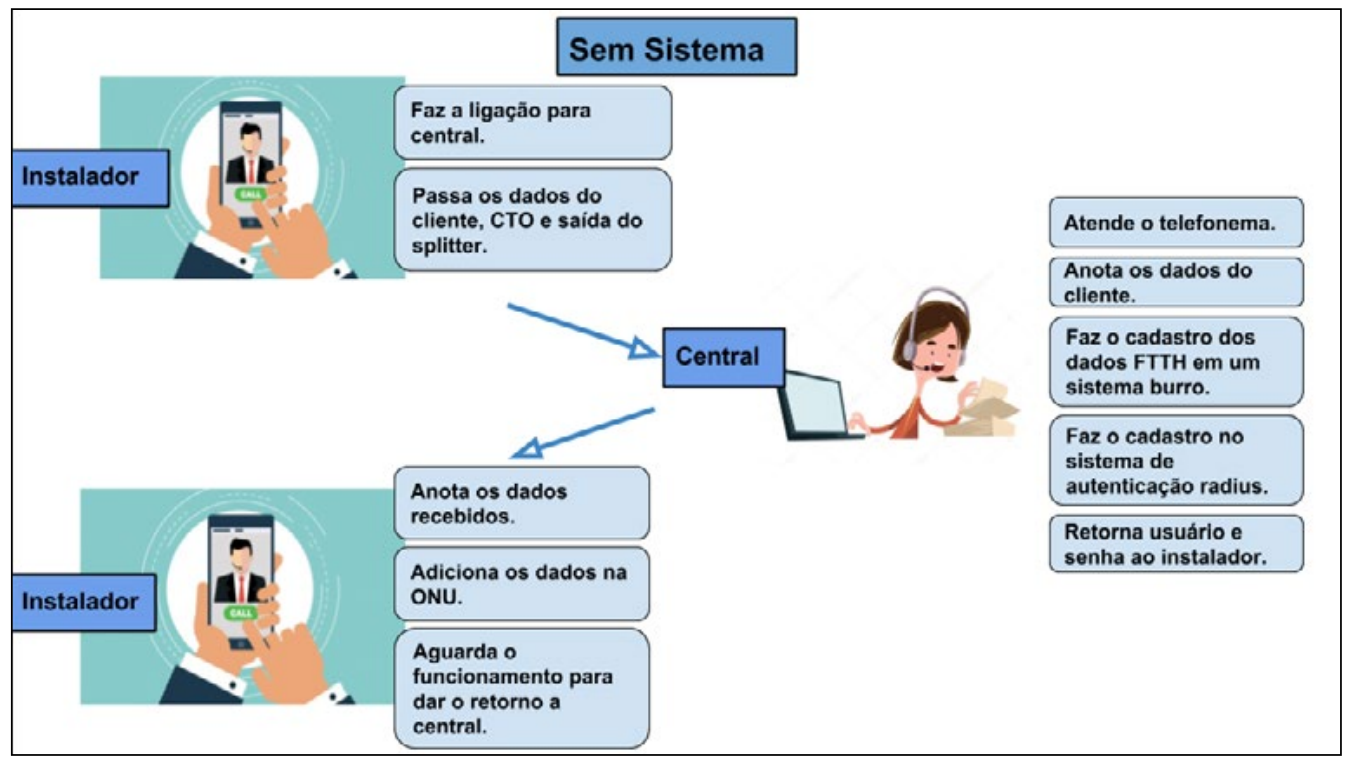

Fonte: Elaborado pelo autor (2017).

A Figura 7 mostra como é feito o cadastro do cliente com o sistema desenvolvido, muitas etapas são eliminadas trazendo mais agilidade na instalação e com muitas informações adicionais importantes para gerência e gestão.

Figura 7 - Cadastro do cliente (Com Sistema)

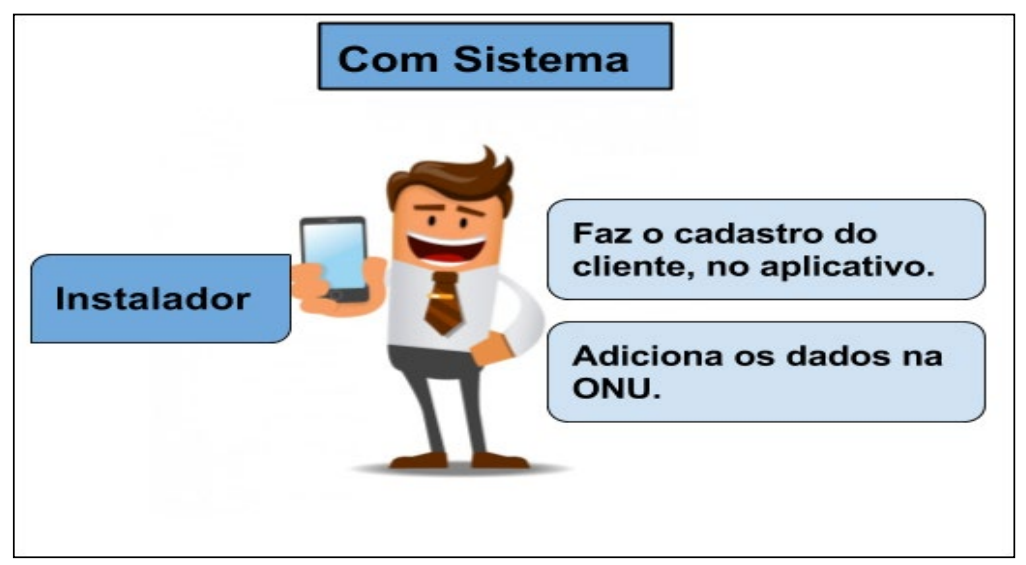

Fonte: Elaborado pelo autor (2017).

A Figura 8 mostra o cadastro de cliente, acessado via aplicativo mobile acessado pelos instaladores da empresa. 
Figura 8 - Tela Cadastro do cliente (Mobile)
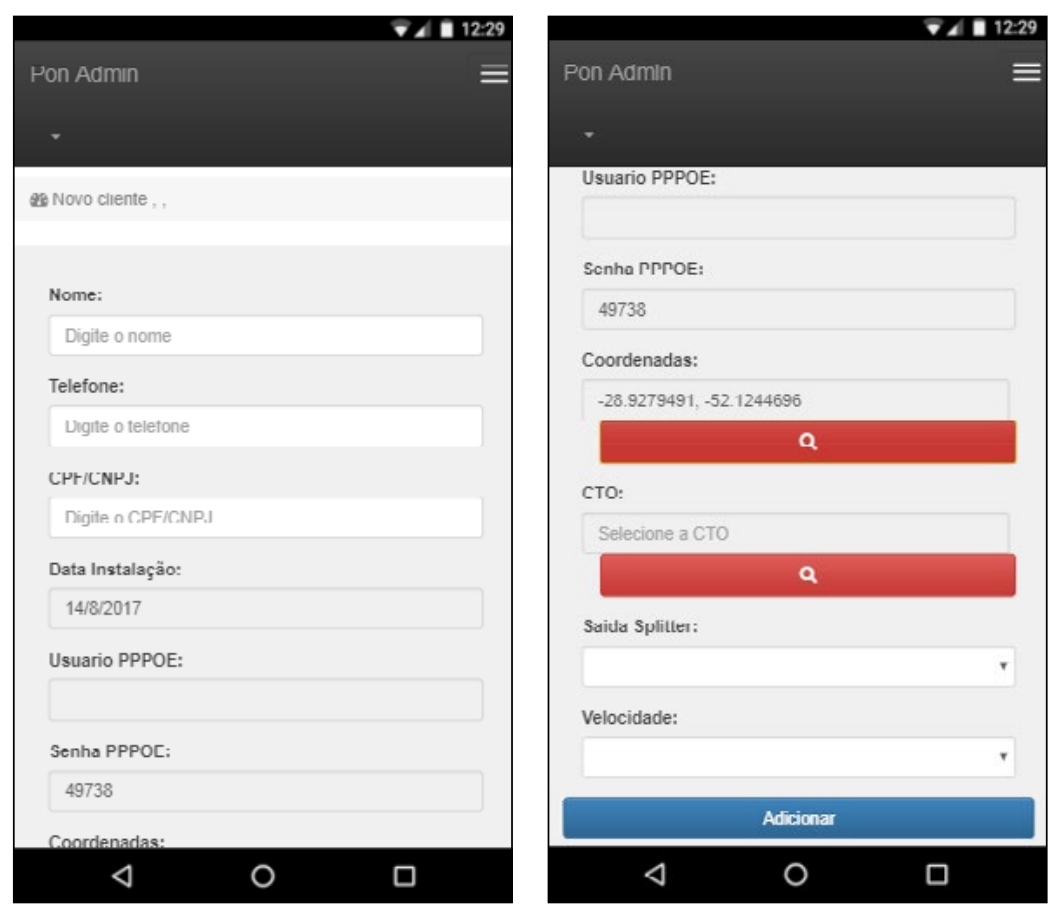

Fonte: Elaborado pelo autor (2017).

\subsection{Dados do Mapa}

O mapa é utilizado tanto no sistema web como no aplicativo mobile, pois é nele que é exibido o local onde estamos e as caixas de atendimento próximas. No mapa também conseguimos visualizar os clientes cadastrados. A Figura 9 mostra o mapa sendo acessado pelo sistema WEB. 
Figura 9 - Mapa exibido no Sistema WEB

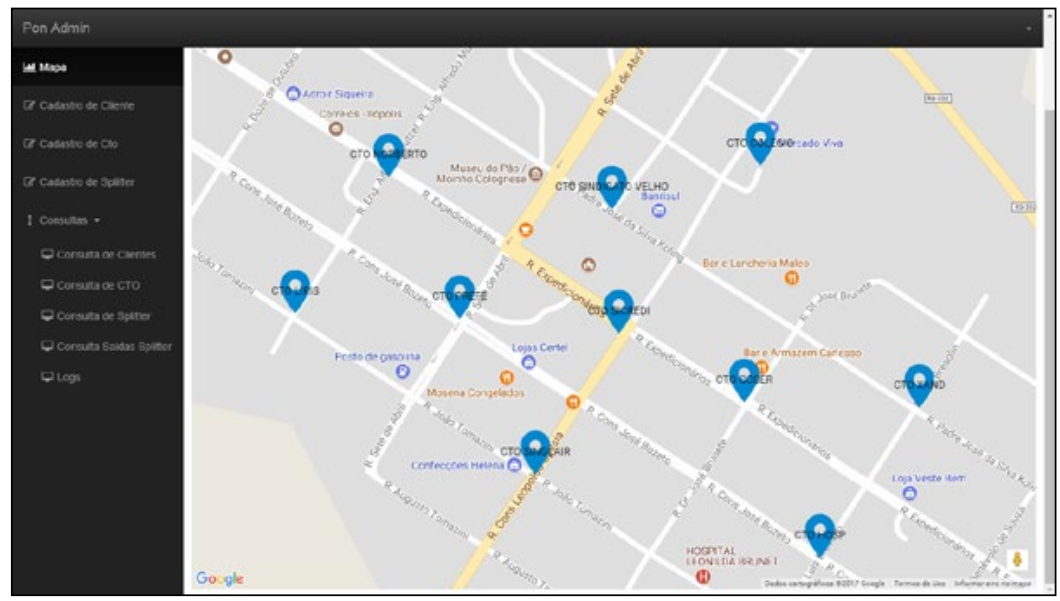

Fonte: Elaborado pelo autor (2017).

A Figura 10(A) mostra as camadas de visualização que o sistema possui, podendo exibir somente as CTOs, somente os clientes ou todos eles juntos, a imagem 10(B) mostra como é visualizado no mapa as informações da CTO, essa tabela é exibida ao clicar na caixa de atendimento, ela exibe quantas saídas o splitter que está instalado na caixa de atendimento possui e se há cliente instalado em cada saída.

Figura 10 - Camadas de visualização (A) e Visualização da CTO (B)
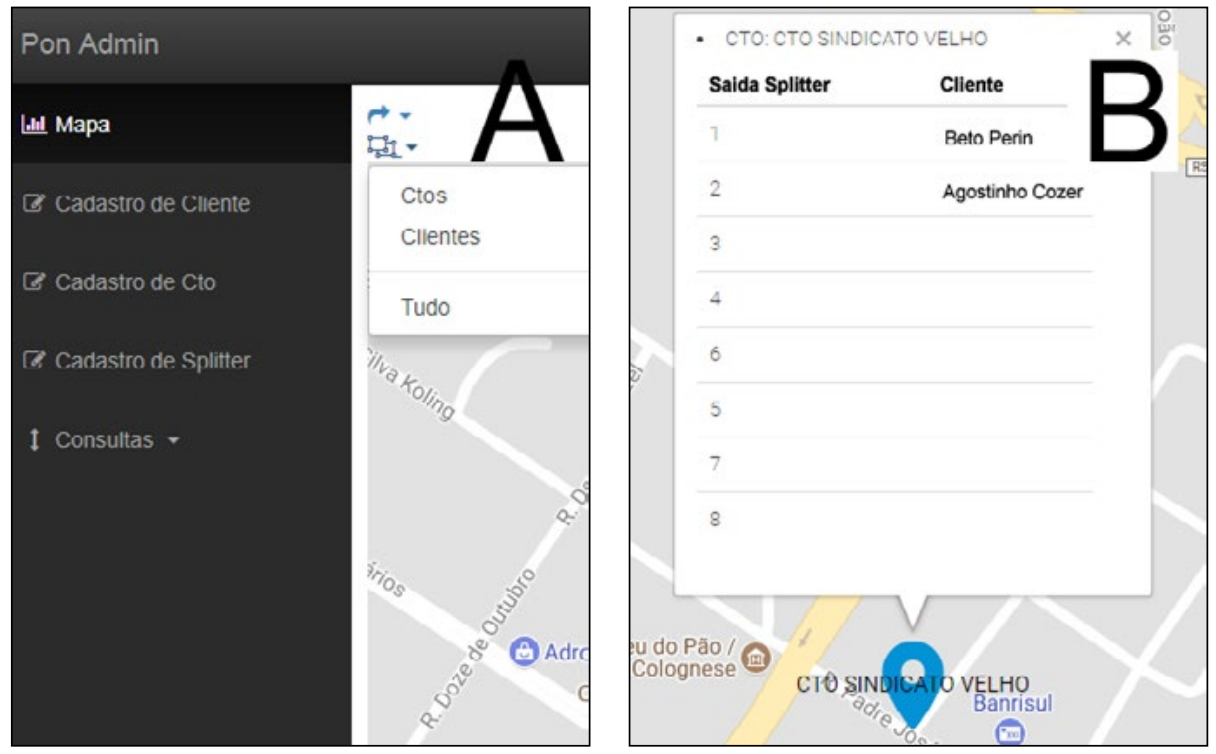

Fonte: Elaborado pelo autor (2017). 
Uma das vantagens de utilizar o aplicativo é a facilidade de reconhecimento da rede, a Figura 11 exibe o mapa aberto no aplicativo mobile, nele são exibidas as caixas de atendimento próximas ao instalador, a posição do instalador é obtida pelas coordenadas do dispositivo. Na tela, o instalador está com o marcador em vermelho, enquanto as caixas de atendimento mostram em azul.

Figura 11 - Visualização das caixas de atendimento próximas

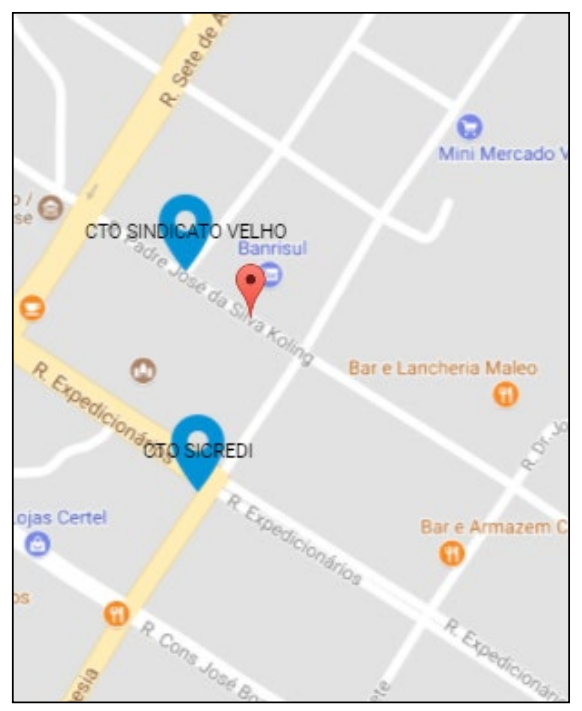

Fonte: Elaborado pelo autor (2017).

\subsection{Consulta de Clientes}

A consulta de clientes traz informações dos clientes, geralmente utilizado para alguma manutenção pós-instalação. A Figura 12 mostra como os dados são exibidos no sistema. 
Figura 12 - Consulta de clientes no sistema

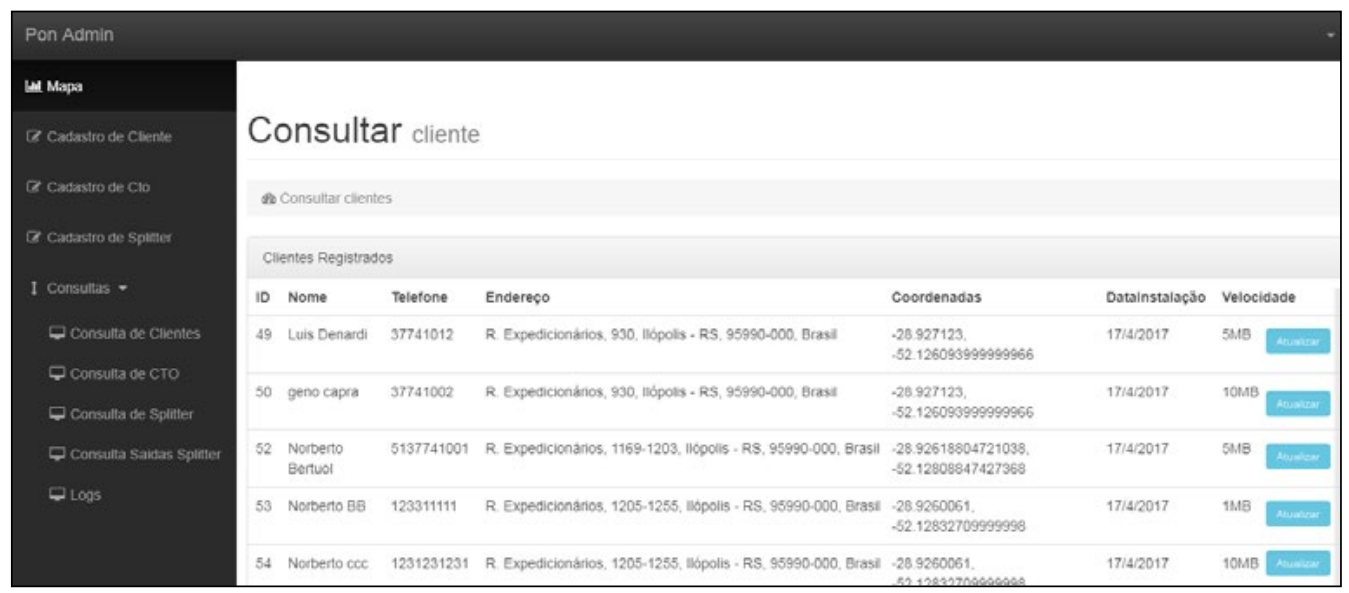

Fonte: Elaborado pelo autor (2017).

Esta seção apresentou a estrutura e funcionalidades do sistema desenvolvido. A seguir serão analisados dados e resultados dos testes feitos após sua implantação em uma empresa de telecomunicações.

\section{RESULTADOS E DISCUSSÃO}

Esta seção apresenta os resultados obtidos após o desenvolvimento do sistema e sua implantação em uma empresa de telecomunicações, os resultados foram obtidos com base em entrevistas elaboradas pelo autor após duas semanas de testes, os utilizadores do sistema foram três usuários instaladores e um usuário como permissão de gerência.

Após o período de utilização foi feito uma entrevista com os usuários do aplicativo sobre a usabilidade e utilidade da ferramenta os seus pontos positivos e negativos, as sugestões e melhorias, e os resultados encontram-se a seguir.

a) Pontos Positivos

- Facilidade de inclusão de novos clientes;

- Ótima visualização das CTOs e clientes no mapa;

- Fácil interação com menus e janelas;

- Preenchimento automático de vários campos;

- Praticidade nas consultas via smartphone;

- Todos os dados do cliente e conexão no mesmo local.

b) Pontos Negativos

- Dificuldade de selecionar o local no smartphone sem GPS; 
- Alguns erros aleatórios geram o travamento do sistema, que precisava ser recarregado.

c) Sugestões e Melhorias

- Criação de Mapa de Cabos;

- Permitir acrescentar uma Foto dos postes onde estão as CTOs;

- Integrar com o sistema de cobrança;

- Integrar com o sistema de ordens de serviço.

Os resultados obtidos com a avaliação do sistema desenvolvido são exibidos no Gráfico 1, que demonstra a opinião dos utilizadores sobre o software, quando foram perguntados: "Na sua avaliação, o sistema atende os requisitos e necessidades de um provedor em relação a gerência e administração das redes FTTH?"

Gráfico 1 - Gráfico das avaliações do sistema

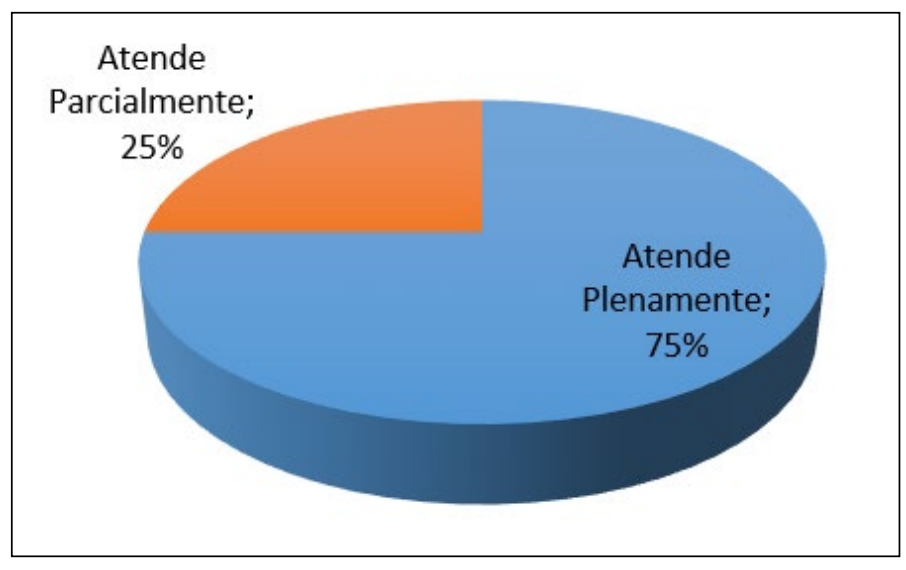

Fonte: Elaborado pelo autor (2017).

Pode-se perceber que o software conseguiu suprir as necessidades básicas do provedor, levando em consideração que o aplicativo está em fase de testes, e foi bem aceito pelos funcionários do provedor, sendo que suas opiniões ajudaram na implementação de novas funcionalidades e melhorias.

Quando aos pontos negativos, em relação ao travamento do aplicativo, foi verificado que só ocorria nos navegadores de internet com versões desatualizadas, utilizando um navegador atualizado não foi detectado nenhuma falha.

\section{CONSIDERAÇÕES FINAIS}

Neste trabalho realizou-se uma breve abordagem sobre a evolução dos meios de comunicação utilizados para transmissão de internet, apresentando 
o cenário atual, que registra um aumento considerável do uso da comunicação por fibra óptica FTTH, devido às vantagens apresentadas.

Foram analisadas características que influenciam a implementação da tecnologia de redes ópticas passivas, no contexto de redes de fibras ópticas até as residências dos usuários. Nos próximos anos espera-se que essa tecnologia esteja presente em muitas residências e pontos comerciais e, portanto, é de extrema importância que os provedores utilizem ferramentas para gerência e documentação destas redes, para posterior manutenção e facilidade nos controles necessários.

Então, frente a essa a necessidade da gerência destas redes ópticas, pois ao longo do tempo, se não houver uma boa documentação ela se torna complexa de administrar, propôs-se o desenvolvimento de um sistema de gerência para redes PONT (FTTH), para atender as necessidades de um provedor local.

De acordo com os resultados obtidos conclui-se que o sistema se apresentou suficiente para gerência e gestão da rede FTTH do provedor para o qual a solução foi desenvolvida, pois além de agilizar a instalação dos clientes ele dá suporte ao instalador e ao gestor da empresa, otimizando processos e facilitando a administração da rede óptica.

Após o desenvolvimento do trabalho foram identificadas novas funcionalidades que podem fazer parte de trabalhos futuros, dentre elas estão:

- Integração com o sistema de cobrança;

- Módulo de ordens de serviço;

- Calcular e perspectiva de luz chegando ao assinante;

- Exibir os mapas de cabos.

Ao final deste trabalho, conclui-se que os objetivos propostos foram cumpridos com êxito. $\mathrm{O}$ autor pretende continuar com o desenvolvimento da ferramenta e talvez disponibilizar a solução no mercado, para que a mesma possa atender e auxiliar outras empresas da área.

\section{REFERÊNCIAS}

ANGULARJS. Guide to AngularJS Documentation. Google Inc. 2017. Disponível em: <https://docs.angularjs.org/guide/concepts>. Acesso em: 28 abr. 2017.

CHEMIN, Beatris Francisca. Manual da Univates para trabalhos acadêmicos: planejamento, elaboração e apresentação. 3. ed. Lajeado: Univates, 2015.

CORDOVA. Apache Cordova. Disponível em <https://cordova.apache.org/>. Acesso em: 21 mai. 2017.

COSTA, Carlos J. Desenvolvimento para Web. Portugal: Lusocrédito, 2007. 
FERRARO, Richard; AKTIHANOGLU, Murat. Location-Aware Applications. Shelter Island: Manning Publications, 2011.

FRENZEL, Louis E. Fundamentos de Comunicação Eletrônica. 3. ed. São Paulo: AMGH. 2013.

FURUKAWA. Furukawa Electric Brasil. Disponível em <www.furukawa.com.br $>$. Acesso em: 14 mai. 2017.

GIL, Antônio Carlos. Como elaborar projetos de pesquisa. 4. ed. São Paulo: Atlas, 2010.

GOOGLE. Google Android. API do Google Android. Disponível em: <https:/ / developer.android.com/studio>. Acesso em: 27 mar. 2017.

GOOGLE. Google Maps. API do Google Maps. Disponível em: < https:/ / developers. google.com/maps/documentation>. Acesso em: 23 mar. 2017.

KEISER, Gerd. Comunicações por fibras ópticas. 4. ed. Porto Alegre: AMGH, 2014.

KITAYAMA, Ken-ichi; 口MASETTI-PLACCI, Francesco; PRATI, qGiancarlo. Optical Networks and Technologies.1. ed. USA: SPRINGER, 2006.

ITU-T. G.984.1: Gigabit-capable passive optical networks (GPON): General characteristics. 2008. Disponível em: <https:/ / www.itu.int/rec/T-REC-G.984.1>. Acesso em: 20 mar. 2017.

LAFATA, P. Advanced Algorithm for Optimizing the Deployment Cost of Passive Optical Networks, Prague, 11, n. 1, Março, 2013. 10p.

LECHETA, Ricardo R. Google ANDROID Aprenda a criar aplicações para dispositivos móveis com Android SDK. 3. ed. São Paulo: Novatec, 2014.

LEVAY, Leonardo S. Ferramenta para Projetos de Redes Ópticas Passivas PON. Trabalho de Conclusão de Curso Em Engenharia Elétrica, 2013, Universidade de Brasília, Brasíla, DF, 76p.

MARIN, Paulo Sérgio. Cabeamento Estruturado. 5. ed. São Paulo: ÉRICA, 2015.

SESHADRI, Shyam; GREEN, Brad. Desenvolvendo com AngularJS: aumento de produtividade com aplicações web estruturadas. São Paulo: Novatec, 2008.

SPURLOCK, Jake. Bootstrap. 1. ed. Sebastopol, CA. O’Really Media, 2013. 